\title{
Novel in vitro model for studying ureteric stent-induced cell injury
}

Elwood CN, Lange D, Nadeau R, Seney S, Summers K, Chew BH, Denstedt JD, Cadieux PA

Lawson Health Research Institute, University of Western Ontario, London, Canada.

BJU Int. 2010; 105: 1318-23

Objective: To develop a novel in vitro model for the study of bladder and kidney epithelial cell injury akin to stent movement, as ureteric stents are associated with urinary tract complications that can significantly add to patient morbidity. These sequelae may be linked to inflammation triggered by stent-mediated mechanical injury to the urinary tract.

Materials and Methods: T24 bladder and A498 kidney cell line monolayers were damaged mechanically by segments of either Percuflex Plus (PP) or Triumph (triclosan-eluting) stents (both from Boston Scientific Corporation Inc. Natick, MA, USA) and the resulting expression profiles of several pro-inflammatory cytokines and growth factors were analysed.

Results: After control injury using the PP stent, supernatants of both cell lines had significantly increased levels of interleukin (IL)-6, IL-8, basic fibroblast growth factor and platelet-derived growth factor BB, and A498 cells also had increased tumour necrosis factor alpha. In almost all cases, the presence of triclosan within the media abrogated the pro-inflammatory cytokine increases, while its effects on growth factors varied.

Conclusion: This study suggests that stent-related symptoms in the bladder and kidney may be partially due to a local inflammatory response to epithelial damage caused by the presence and movement of the stent. Future stent design should take these inflammatory responses, with respect to physical injury, into consideration, using either more biocompatible materials or anti-inflammatory compounds such as triclosan.

\section{Editorial Comment}

The authors have previously evaluated ketorolac coated stents - noting no significant improvement in patient symptoms. It would be of value to test the anti-inflammatory properties of ketorolac-coated stents in this novel in vitro model. It would be interesting to develop epithelial: smooth muscle co-cultured matrices to evaluate the impact of stromal: epithelial interactions following stent irritation on the expression of inflammatory markers and growth factors. The concept of uroepithelial cell disruption as a cause for stent pain may suggest that those patients undergoing long ureteroscopic procedures with forceful irrigation may experience more stent discomfort due to the hydrodistension of the upper collecting system and subsequent stimulation of the inflammatory response. It would be important to evaluate inflammatory markers in urine after ureteroscopy and with urinary stents.

\author{
Dr. Manoj Monga \\ Department of Urology \\ Cleveland Clinic Foundation \\ Cleveland, Ohio, USA \\ E-mail: endourol@yahoo.com
}

